PROCEEDINGS OF THE

AMERICAN MATHEMATICAL SOCIETY

Volume 140, Number 7, July 2012, Pages 2193-2207

S 0002-9939(2011)11072-4

Article electronically published on October 24, 2011

\title{
SPANNING AND INDEPENDENCE PROPERTIES OF FRAME PARTITIONS
}

\author{
BERNHARD G. BODMANN, PETER G. CASAZZA, VERN I. PAULSEN, \\ AND DARRIN SPEEGLE
}

(Communicated by Thomas Schlumprecht)

\begin{abstract}
We answer a number of open problems in frame theory concerning the decomposition of frames into linearly independent and/or spanning sets. We prove that Parseval frames with norms bounded away from 1 can be decomposed into a number of sets whose complements are spanning, where the number of these sets only depends on the norm bound. Further, we prove a stronger result for Parseval frames whose norms are uniformly small, which shows that in addition to the spanning property, the sets can be chosen to be independent and the complement of each set can contain a number of disjoint, spanning sets.
\end{abstract}

\section{INTRODUCTION}

Over the last few decades, frame theory has developed into a vibrant subject including contributions in time-frequency analysis [9, 19, 13, 10, 8, 11, and applications in engineering such as wireless communications or other types of signal and image processing techniques; see the survey papers [15, 16] and the many references therein. In pure mathematics, frame theory has opened up new approaches to one of the significant open problems in analysis today - the notoriously intractable 1959 Kadison-Singer Problem [3, 7, 6].

Formally, a frame is a family of vectors $\left\{f_{i}\right\}_{i \in I}$ in a real or complex Hilbert space $\mathbb{H}$ so that there are constants $0<A \leq B<\infty$ (called the lower and upper frame bounds, respectively) satisfying

$$
A\|x\|^{2} \leq \sum_{i \in I}\left|\left\langle x, f_{i}\right\rangle\right|^{2} \leq B\|x\|^{2}, \text { for all } x \in \mathbb{H} .
$$

The frame is an $A$-tight frame if $A=B$, a Parseval frame if $A=B=1$, an equal norm frame if $\left\|f_{i}\right\|=c$, for all $i \in I$, and a unit norm frame if $c=1$. If we only require the upper frame bound $B$, we call this a $B$-Bessel sequence. Associated to a frame we have the analysis operator $T: \mathbb{H} \rightarrow \ell_{2}(I)$ given by $T x=\sum_{i \in I}\left\langle x, f_{i}\right\rangle e_{i}$, where $\left\{e_{i}\right\}_{i \in I}$ is the natural orthonormal basis of $\ell_{2}(I)$. Its

Received by the editors October 29, 2010 and, in revised form, February 14, 2011.

2010 Mathematics Subject Classification. Primary 15A03, 42C15.

The first author was supported by NSF grant DMS-0807399.

The second author was supported by NSF 1008183: DTRA/NSF 1042701.

The third author was supported by NSF DMS-0600191.

The fourth author was supported by NSF DMS-0354957.

(C)2011 American Mathematical Society Reverts to public domain 28 years from publication 
adjoint $T^{*}$ is the synthesis operator given by $T\left(\sum_{i \in I} a_{i} e_{i}\right)=\sum_{i \in I} a_{i} f_{i}$. The frame operator $S=T^{*} T$ satisfies $S x=\sum_{i \in I}\left\langle x, f_{i}\right\rangle f_{i}$ for $x \in \mathbb{H}$. It provides the reconstruction formula

$$
x=\sum_{i \in I}\left\langle x, S^{-1} f_{i}\right\rangle f_{i}, \text { for all } x \in \mathbb{H} .
$$

We refer the reader to $[8$ for the requisite background in frame theory.

What makes frames so useful in practice is their redundancy. That is, in general a frame might have smaller subsets, each with a dense linear span in the space $\mathbb{H}$. Accordingly, every vector in the space has uncountably many representations with respect to a frame, not just the canonical choice given by equation (1.2). The flexibility in choosing representations is key to many applications [15, 16. One of the most important problems in frame theory today is therefore to understand redundancy and its role in these applications. This includes a long list of fundamental questions concerning the behavior of subsets of a frame such as how many (disjoint) spanning sets does a frame contain? Or, how many (disjoint) linearly independent subsets does it contain? Or, putting these together, how many disjoint linearly independent spanning sets does our frame contain? Can we partition any unit norm frame into a finite number of subsets with specialized properties so that each subset is nearly tight for its span, i.e. with $\frac{B}{A} \approx 1$ ? This innocent looking problem in frame theory is now known to be equivalent to the 1959 Kadison-Singer Problem [4, 7.

One of the first results concerning the decomposition of frames into linearly independent sets is in 3], where it is shown that a frame can be partitioned into $\lceil B\rceil$-linearly independent sets. In 7$]$ it is shown that a unit norm tight frame $\left\{f_{i}\right\}_{i=1}^{K M}$ in an $M$-dimensional space can be partitioned into $K$ linearly independent spanning sets. It has been an open question since [7] appeared whether there is a similar result for unit norm frames $\left\{f_{i}\right\}_{i=1}^{K M+r}$ for $0<r<M$. This is one of the questions we will answer in this paper. Recently, an intuitive, quantitative measure for redundancy was given for finite frames [2]. It is interesting to note that it relates to independence and spanning properties of frame partitions which we explore here. We expect that such a systematic refinement of our understanding of redundancy will have an important impact on applications.

Underlying much of this paper is the classification theorem for Parseval frames (see e.g. [8]):

Theorem 1.1 (Classification theorem for Parseval frames). Let $\left\{f_{i}\right\}_{i \in I}$ be a frame for $\mathbb{H}$ with analysis operator $T$. Then $\left\{f_{i}\right\}_{i \in I}$ is a Parseval frame for $\mathbb{H}$ if and only if $T$ is an isometry and the orthogonal projection $P: \ell_{2}(I) \rightarrow T(\mathbb{H})$ satisfies $T f_{i}=P e_{i}$, for all $i \in I$. In particular, this projection is given by the Gram matrix $\left(\left\langle f_{j}, f_{i}\right\rangle\right)_{i, j \in I}$.

Moreover, in this case, $\left\{(I-P) e_{i}\right\}_{i \in I}$ is a Parseval frame for its span, called the Naimark Complement of $\left\{f_{i}\right\}_{i \in I}$.

It is immediate that an orthogonal projection takes a frame with frame bounds $A, B$ to a frame for its range with the same frame bounds. In particular, an orthogonal projection maps a Parseval frame to a Parseval frame.

We will concentrate on Parseval frames here since with respect to independence and spanning properties, this is really the general case in the sense that every frame $\left\{f_{i}\right\}_{i \in I}$ is isomorphic to the Parseval frame $\left\{S^{-1 / 2} f_{i}\right\}_{i \in I}$, where $S$ is the 
frame operator. That is, $S^{-1 / 2}$ is an invertible operator mapping our frame to a Parseval frame, and hence it maintains linearly independent properties, spanning properties and Riesz basic sequences. Many of our results rely on the assumption that the norms of the Parseval frame vectors are uniformly bounded away from 1. This is a necessary assumption since for a Parseval frame $\left\{f_{i}\right\}_{i \in I}$, equation (1.1) quickly yields that if $\left\|f_{j}\right\|=1$, then $f_{j} \perp$ span $\left\{f_{i}\right\}_{i \neq j}$.

The remainder of this paper is organized as follows: In Section 2, we will give a dichotomy between linearly independent subsets of a Parseval frame and spanning subsets of its Naimark Complement. This represents a new approach to these questions which previously concentrated only on finding linearly independent subsets of a frame. We will then show that Parseval frames whose vectors have norms uniformly bounded away from one can be partitioned into a finite number of subsets so that the complement of each subset spans the whole space. In Section 3, we will derive a non-trivial variation of the famous Rado-Horn Theorem and use it to show that every finite equal norm Parseval frame can be partitioned into linearly independent spanning sets with (perhaps) a leftover linearly independent set. This answers a problem stemming from the results in [7].

\section{SPANNING PROPERTIES FOR FRAME PARTITIONS}

In Proposition 2.3 we establish a dichotomy between independence properties of subsets of a finite Parseval frame and spanning properties of complementary subsets of its Naimark Complement. This gives a new approach to the Kadison-Singer Problem which is complementary to the standard equivalences of the problem. In Theorem 2.8 we will apply this result to show that, given a Parseval frame $\left\{f_{n}\right\}_{n \in \mathbb{N}}$ with $\left\|f_{n}\right\|^{2} \leq 1-\delta$, we can partition $\mathbb{N}$ into $r$-subsets ( $r$ only depending upon $\delta$ ) $\left\{A_{j}\right\}_{j=1}^{r}$ so that span $\left\{f_{n}\right\}_{n \in A_{k}^{c}}=\mathbb{H}$, for all $1 \leq k \leq r$.

To establish our dichotomy, we will first classify when submatrices of the Gram matrix of a Parseval frame are one-to-one operators. For notation, given a family of vectors $\mathcal{F}=\left\{f_{i}\right\}_{i \in S}$ in a Hilbert space $\mathbb{H}$, where $S$ is some index set and a subset $B \subseteq S$, we write $\mathcal{F}_{B}=\left\{f_{i}\right\}_{i \in B}$ and let $\mathbb{H}_{B}$ denote the closed linear span of $\mathcal{F}_{B}$. Recall that by Theorem 1.1 and the discussion following it, orthogonal projections take Parseval frames to Parseval frames and $\left\{f_{i}\right\}_{i \in S}$ is a Parseval frame for $\mathbb{H}$ if and only if the Gram matrix $G=\left(\left\langle f_{j}, f_{i}\right\rangle\right)_{i, j \in S}$ is the matrix of a projection operator on $\ell^{2}(S)$.

We start with a simple decomposition of Gram matrices.

Proposition 2.1. Let $\left\{f_{i}\right\}_{i \in S}$ be a Parseval frame for $\mathbb{H}$, let $P$ be an orthogonal projection onto a closed subspace of $\mathbb{H}$ and let $I$ denote the identity operator on $\mathbb{H}$. Then $G=\left(\left\langle f_{j}, f_{i}\right\rangle\right)_{i, j, \in S}, R=\left(\left\langle P f_{j}, P f_{i}\right\rangle\right)_{i, j \in S}$ and $Q=\left(\left\langle(I-P) f_{j}\right.\right.$, $\left.\left.(I-P) f_{i}\right\rangle\right)_{i, j \in S}$ are the matrices of projection operators on $\ell^{2}(S)$ with $G=R+Q$. Moreover, $P=I$ if and only if 1 is not an eigenvalue of $Q$.

Proof. The equality $G=R+Q$ is immediate from $R=\left(\left\langle P f_{j}, f_{i}\right\rangle\right)$ and $Q=$ $\left(\left\langle(I-P) f_{j}, f_{i}\right\rangle\right)$ for each $i, j \in S$, and from the linearity of the inner product in the first entry. The fact that $G, Q$ and $R$ are matrices of projections follows from the fact that the vectors $\left\{f_{i}\right\}_{i \in S}$ form a Parseval frame and the above remarks.

For the final statement, note that $P=I$ if and only if $Q=0$. However, since $Q$ is a projection, $Q=0$ if and only if 1 is not an eigenvalue. 
Given a subset $B \subseteq S$, we let $D_{B}=\left(d_{i, j}\right)_{i, j \in S}$ denote the bounded operator on $\ell^{2}(S)$ whose matrix is the diagonal matrix with $d_{i, i}=1$ when $i \in B$ and $d_{i, j}=0$ when $i \in B^{c}$ or $j \in B^{c}$, the complement of the set $B$. Now we will relate the spanning properties of a subset of a Parseval frame to 1 not being an eigenvalue of the corresponding submatrix of the Gram matrix.

Proposition 2.2. Let $\left\{f_{i}\right\}_{i \in S}$ be a Parseval frame for $\mathbb{H}$, let $G=\left(\left\langle f_{j}, f_{i}\right\rangle\right)_{i, j \in S}$ denote its Gram matrix, and let $B \subseteq S$. Then $\mathbb{H}_{B}=\mathbb{H}$ if and only if 1 is not an eigenvalue of $D_{B^{c}} G D_{B^{c}}$.

Proof. Let $P$ denote the projection onto $\mathbb{H}_{B}$ and apply Proposition 2.1. Since for $j \in B, f_{j} \in \mathbb{H}_{B}$, we have that when $j \in B$, then $\left\langle f_{j}, f_{i}\right\rangle=\left\langle P f_{j}, P f_{i}\right\rangle$. More generally, the matrices $G$ and $R$ are equal in any entry $(i, j)$ provided that $i \in B$ or $j \in B$. Thus, the matrix $Q$ must be 0 in any such entry. Hence we obtain the operator inequalities $0 \leq Q=D_{B^{c}} Q D_{B^{c}} \leq D_{B^{c}} G D_{B^{c}} \leq D_{B^{c}}$.

Now, if 1 is not an eigenvalue of $D_{B^{c}} G D_{B^{c}}$, then these inequalities imply that 1 is not an eigenvalue of $Q$. Invoking the preceding proposition, we get $P=I$, and so $\mathbb{H}_{B}=\mathbb{H}$.

Conversely, assume that 1 is an eigenvalue of $D_{B^{c}} G D_{B^{c}}$. Write $G=V V^{*}$, where $V: \mathbb{H} \rightarrow \ell^{2}(S)$ is the analysis operator of the Parseval frame. Since $D_{B^{c}} G D_{B^{c}}=\left(V^{*} D_{B^{c}}\right)^{*}\left(V^{*} D_{B^{c}}\right)$, we have that $\left(V^{*} D_{B^{c}}\right)\left(V^{*} D_{B^{c}}\right)^{*}=V^{*} D_{B^{c}} V$ also has eigenvalue 1. By the Parseval property, $V$ is an isometry, $V^{*} V=I$, and necessarily $V^{*} D_{B} V=I-V^{*} D_{B^{c}} V$ has eigenvalue zero. Thus the range of $V^{*} D_{B}$ is orthogonal to the corresponding eigenvectors. Since the closure of the range of $V^{*} D_{B}$ is by definition $\mathbb{H}_{B}$, it is not equal to $\mathbb{H}$.

Now we can give our complementarity principle between spanning and linear independence.

Proposition 2.3. Let $\mathbb{H}$ be a Hilbert space with orthonormal basis $\left\{e_{j}\right\}_{j \in S}$, let $P$ be the orthogonal projection onto a closed subspace of $\mathbb{H}$, and let $B \subseteq S$. Then the linear span of $\left\{P e_{j}\right\}_{j \in B}$ is dense in $P(\mathbb{H})$ if and only if the operator $\left(\left\langle(I-P) e_{j},(I-P) e_{i}\right\rangle\right)_{i, j \in B^{c}}$ on $\ell^{2}\left(B^{c}\right)$ is one-to-one.

Proof. Note that the set $\left\{P e_{j}: j \in S\right\}$ is a Parseval frame for $P(\mathbb{H})$. Hence, the span of $\left\{P e_{j}: j \in B\right\}$ is dense in $P(\mathbb{H})$ if and only if the matrix $Q=\left(\left\langle P e_{j}, P e_{i}\right\rangle\right)_{i, j \in B_{c}}$ does not have 1 as an eigenvalue. However, since

$$
I_{\ell^{2}\left(B^{c}\right)}-Q=\left(\left\langle(I-P) e_{j},(I-P) e_{i}\right\rangle\right)_{i, j \in B^{c}},
$$

$Q$ not having eigenvalue 1 is equivalent to the latter matrix having a trivial kernel.

Recall that a family of vectors $\left\{f_{i}\right\}_{i \in I}$ is called an $\ell_{2}$-independent set if whenever $\left\{a_{i}\right\}_{i \in I} \in \ell_{2}(I)$ and $\sum_{i \in I} a_{i} f_{i}=0$, then $a_{i}=0$ for all $i \in I$. In this language, Proposition 2.3 becomes:

Corollary 2.4. Let $\mathbb{H}$ be a Hilbert space with orthonormal basis $\left\{e_{j}\right\}_{j \in S}$, let $P$ be the orthogonal projection onto a closed subspace of $\mathbb{H}$, and let $B \subseteq S$. Then the linear span of $\left\{P e_{j}\right\}_{j \in B}$ is dense in $P(\mathbb{H})$ if and only if $\left\{(I-P) e_{i}\right\}_{i \in B^{c}}$ is $\ell_{2}$-independent. 
Proof. The operator $A=\left(\left\langle(I-P) e_{j},(I-P) e_{i}\right\rangle\right)_{i, j \in B^{c}}$ satisfies for all $a=\left\{a_{i}\right\}_{i \in B^{c}} \in$ $\ell_{2}\left(B^{c}\right)$ that

$$
A(a)=\left(\left\langle(I-P) e_{j}, \sum_{i \in B^{c}} a_{i}(I-P) e_{i}\right\rangle\right)_{i, j \in B^{c}} .
$$

In particular, $\sum_{i \in B^{c}} a_{i}(I-P) e_{i}=0$ if and only if $A(a)=0$ if and only if $a=0$.

In the finite dimensional setting, Proposition 2.3 implies:

Corollary 2.5. If $P$ is an orthogonal projection on a finite dimensional Hilbert space $\mathbb{H}$, then $\left\{P e_{j}\right\}_{j \in B}$ spans $P(\mathbb{H})$ if and only if the set $\left\{(I-P) e_{j}: j \in B^{c}\right\}$ is linearly independent.

For the final results in this section, we will show how to partition a Parseval frame whose vectors are uniformly bounded away from 1 in norm into subsets whose complementary sets are spanning.

For these results, we will need two results from the literature which involve partitioning frames into linearly independent sets or into $\ell_{2}$-independent sets. The first is due to Casazza, Christensen, Lindner and Vershynin [3].

Theorem 2.6. If $\left\{f_{n}\right\}_{n \in \mathbb{N}}$ is a unit norm B-Bessel sequence, then $\left\{f_{n}\right\}_{n \in \mathbb{N}}$ can be partitioned into $r=\lceil B\rceil$ linearly independent sets.

We also need the following result of Casazza, Kutyniok, Speegle and Tremain 6].

Theorem 2.7. Every unit norm Bessel sequence which is finitely linearly independent is a union of two $\ell_{2}$-independent sets.

Theorem 2.8. Let $0<\delta<1$, and set $r=2\left\lceil\frac{1}{\delta}\right\rceil$. If $\left\{f_{n}\right\}_{n \in \mathbb{N}}$ is a Parseval frame for a Hilbert space $\mathbb{H}$, with $\left\|f_{n}\right\|^{2} \leq 1-\delta$ for all $n \in \mathbb{N}$, then there exists a partition of $\mathbb{N}$ into $r$ disjoint sets, $A_{1} \cup \cdots \cup A_{r}=\mathbb{N}$, such that $\mathbb{H}_{A_{k}^{c}}=\mathbb{H}$, for $k=1, \ldots, r$.

Proof. By the classification theorem for Parseval frames, we may assume that there is a projection $P$ on $\ell_{2}(\mathbb{N})$ satisfying $P e_{n}=f_{n}$ for all $n \in \mathbb{N}$, with $\left\{e_{n}\right\}_{n \in \mathbb{N}}$ the natural orthonormal basis of $\ell_{2}(\mathbb{N})$. By our assumption in the theorem, $\left\|(I-P) e_{n}\right\|^{2}=1-\left\|P e_{n}\right\|^{2} \geq \delta$. It follows that $\left\{\frac{1}{\left\|(I-P) e_{n}\right\|}(I-P) e_{n}\right\}_{n=1}^{\infty}$ is an $\frac{r}{2}$-Bessel sequence. By Theorem 2.6, there is a partition $\left\{B_{1}, B_{2}, \ldots, B_{\frac{r}{2}}\right\}$ of $\mathbb{N}$ so that $\left\{(I-P) e_{n} /\left\|(I-P) e_{n}\right\|\right\}_{n \in B_{j}}$ is finitely linearly independent for all $j \in$ $\left\{1,2, \ldots, \frac{r}{2}\right\}$. Now, applying Theorem [2.7 we can partition each of the $B_{j}$ into two sets with the corresponding vectors $\ell_{2}$-independent. We call $\left\{A_{j}\right\}_{j=1}^{r}$ the resulting partition for $\mathbb{N}$. It follows, of course, that after removing the normalization each $\left\{(I-P) e_{n}\right\}_{n \in A_{j}}$ is also $\ell_{2}$-independent. The theorem now follows from Corollary 2.4 .

Examining the proof of Theorem 2.8 we see that in the finite dimensional case we do not need the extra step of a second partition of the frame into $\ell_{2}$-independent sets. Thus in this case we have:

Theorem 2.9. Let $\delta>0$. Suppose that $\left\{f_{j}: j \in J\right\}$ is a Parseval frame for a finite dimensional Hilbert space with $\left\|f_{j}\right\|^{2} \leq 1-\delta$ for all $j \in J$. Then, it is possible to partition $J$ into $r=\left\lceil\frac{1}{\delta}\right\rceil$ sets $\left\{A_{1}, \ldots, A_{r}\right\}$ such that for each $1 \leq j \leq r$, the family $\left\{f_{i}: i \notin A_{j}\right\}$ spans the space. 
Note that $r$ depends only on the norm bound $1-\delta$, and not the dimension of the space, and an explicit formula for $r$ as a function of $\delta$ is provided. Theorem 2.9 shows the advantage we have gained by switching from working with linearly independent sets to spanning sets. In particular, it is not possible, in general, to get the partition in Theorem 2.9 to have the property that the $\left\{f_{j}\right\}_{j \in A_{i}}$ are linearly independent. The problem is that without a lower bound on the norms of the frame vectors there can be an arbitrarily large number of them. That is, there can be too many frame vectors to be able to partition them into $R$ linearly independent sets for any $R$ which depends only upon $\delta$. However, we will see in the next section that it is possible to achieve a partition in which all sets but one are linearly independent and spanning if the norms of the vectors are uniformly small.

\section{SPANNING AND LINEAR INDEPENDENCE PROPERTIES FOR PARSEVAL FRAMES WITH UNIFORMLY SMALL NORMS}

Until now, we have considered the problem of partitioning Parseval frames into spanning sets or linearly independent sets. Now we will examine the much deeper problem of partitioning a Parseval frame into linearly independent spanning sets. This is a fundamental problem in the field which until now has had only one case that has been answered. Namely, in 7 it is shown that an equal norm Parseval frame $\left\{f_{i}\right\}_{i=1}^{K M}$ for $\mathbb{H}_{M}$ can be partitioned into $K$-linearly independent spanning sets. This proof relies on the Rado-Horn Theorem, and to prove our result, we will have to first strengthen the Rado-Horn Theorem itself. The main theorem in this section is:

Theorem 3.1. Let $\left\{f_{i}\right\}_{i \in I}$ be an equal norm Parseval frame for an $N$ dimensional Hilbert space $\mathbb{H}_{N}$ with $|I|=r N+k$ with $0 \leq k<N$. Then there is a partition $\left\{I_{i}\right\}_{i=1}^{r+1}$ of $I$ so that for $i \in\{2, \ldots, r+1\},\left\{f_{j}\right\}_{j \in I_{i}}$ is a linearly independent spanning set and $\left\{f_{j}\right\}_{j \in I_{1}}$ is linearly independent.

This theorem will follow immediately from Propositions 3.3 and 3.5 combined with Theorem 3.7. Our proof will produce a number of fundamental results giving good bounds on how many linearly independent sets or spanning sets we can divide frames into. Without the equal norm assumption, the theorem fails badly. For example, if $\left\{e_{i}\right\}_{i=1}^{M}$ is an orthonormal basis for $\mathbb{H}_{M}$, the family $f_{1}=\frac{1}{\sqrt{M+1}} e_{1}$, $f_{2}=\frac{1}{\sqrt{M+1}} e_{1}, \ldots, f_{M+1}=\frac{1}{\sqrt{M+1}} e_{1}$, and $f_{j}=e_{j}$ for all $j=M+2, \ldots, 2 M$ is a Parseval frame for $\mathbb{H}_{M}$ with $2 M$-vectors which only contains one linearly independent spanning set, while the theorem would require this to contain 2 linearly independent spanning sets if the vectors had been equal norm. If $r \geq 2$, then this result implies that each set of frame vectors has a complement which is spanning, which was already obtained in Section 3. Moreover, the complement of each set can then be partitioned into at least $r-1$ spanning sets.

Since the proof of Theorem 3.1 is quite long, we will divide it into three subsections as follows:

(1) We first show that a Parseval frame as in Theorem 3.1 can be partitioned into $r+1$ linearly independent sets.

(2) We next show that a Parseval frame as in Theorem 3.1 can be partitioned into $r$ spanning sets. This will follow from some more general results presented in this section which are of independent interest. 
(3) In the last subsection, we show that any frame which has one partition into $r+1$ linearly independent sets and another partition into $r$ linearly independent spanning sets plus a remaining set has a third partition which consists of $r$ linearly independent spanning sets plus a linearly independent set. This result requires adapting the generalized Rado-Horn Theorem given in [5].

3.1. Partitioning a frame into linearly independent sets. In this subsection we will generalize the result of Casazza and Tremain [7] which partitions an equal norm Parseval frame $\left\{f_{i}\right\}_{i=1}^{K M}$ for an $M$ dimensional Hilbert space into $K$ linearly independent spanning sets. For this we need a generalization of the Rado-Horn Theorem due to Casazza, Kutyniok and Speegle [5].

Theorem 3.2. Let $\left\{f_{i}\right\}_{i \in I}$ be a finite collection of vectors in a vector space $X$ and let $M \in \mathbb{N}$. The following conditions are equivalent:

(1) There exists a partition $\left\{I_{j}\right\}_{j=1}^{M}$ of $I$ so that for each $j,\left\{f_{i}\right\}_{i \in I_{j}}$ is linearly independent.

(2) For all $J \subset I$,

$$
\frac{|J|}{\operatorname{dim} \operatorname{span}\left\{f_{i}\right\}_{i \in J}} \leq M
$$

Moreover, in the case that the above conditions fail, there exists a partition $\left\{I_{j}\right\}_{j=1}^{M}$ of $I$ and a subspace $S$ of $X$ such that the following three conditions hold:

(a) For all $1 \leq j \leq M, S=\operatorname{span}\left\{f_{i}: i \in I_{j}\right.$, and $\left.f_{i} \in S\right\}$.

(b) For $J=\left\{i \in I: f_{i} \in S\right\}$

$$
\frac{|J|}{\operatorname{dim} \operatorname{span}\left\{f_{i}\right\}_{i \in J}}>M
$$

(c) For each $1 \leq j \leq M$,

$$
\sum_{i \in I_{j}, f_{i} \notin S} \alpha_{i} f_{i}=0 \text { implies } \alpha_{i}=0, \text { for all } i .
$$

In particular, for each $1 \leq j \leq M,\left\{f_{i}: i \in I_{j}, f_{i} \notin S\right\}$ is linearly independent.

We can now give a generalization of the result of Casazza and Tremain [7] discussed above.

Proposition 3.3. Let $r, k, N$ be natural numbers with $0<k<N$ and let $\left\{f_{i}\right\}_{i=1}^{r N+k}$ be an equal norm Parseval frame for an $N$-dimensional Hilbert space $\mathbb{H}_{N}$. Then $\left\{f_{i}\right\}_{i=1}^{r N+k}$ can be partitioned into $r+1$ linearly independent sets. If $k=0$, then $\left\{f_{i}\right\}_{i=1}^{r N}$ can be partitioned into $r$ linearly independent spanning sets.

Proof. Since $\left\{f_{i}\right\}_{i=1}^{r N+k}$ is an equal norm Parseval frame, we have

$$
N=\sum_{i=1}^{r N+k}\left\|f_{i}\right\|^{2}=(r N+k)\left\|f_{j}\right\|^{2}, \text { for all } j=1,2, \ldots, r N+k .
$$

That is,

$$
\left\|f_{i}\right\|^{2}=\frac{N}{r N+k}, \text { for all } i=1,2, \ldots, N .
$$

We will apply Theorem $3.2(2)$. Choose $J \subset\{1,2, \ldots, r N+k\}$. Let $P$ be the orthogonal projection of $\mathbb{H}_{N}$ onto span $\left\{f_{i}\right\}_{i \in J}$. Since $\left\{P f_{i}\right\}_{i \in J}$ is a Parseval frame 
for its span we have

$$
\operatorname{dim} \operatorname{span}\left\{f_{i}\right\}_{i \in J}=\sum_{i=1}^{r N+k}\left\|P f_{i}\right\|^{2} \geq \sum_{i \in J}\left\|P f_{i}\right\|^{2}=\sum_{i \in J}\left\|f_{i}\right\|^{2}=\frac{N|J|}{r N+k} .
$$

That is,

Hence,

$$
\frac{|J|}{\operatorname{dim} \operatorname{span}\left\{f_{i}\right\}_{i \in J}} \leq \frac{r N+k}{N} .
$$

$$
\frac{|J|}{\operatorname{dimspan}\left\{f_{i}\right\}_{i \in J}} \leq \begin{cases}r & \text { if } k=0, \\ r+1 & \text { if } 0<k<N .\end{cases}
$$

The result now follows by Theorem 3.2 and the fact that in the case $k=0$, we have partitioned an $r N$ element set into $r$ linearly independent sets in an $N$ dimensional Hilbert space $\mathbb{H}_{N}$, and hence each must contain exactly $N$ elements, and so it must be a spanning set.

3.2. Partitioning a frame into spanning sets. In this section we will give fairly accurate bounds on how many spanning sets we can divide a frame into in terms of the lower frame bound of the frame and the norms of the frame vectors. As a useful tool in this work, we first show that a frame which can be partitioned into $r$ linearly independent sets or into $r$ spanning sets has the property that any partition of the frame into $r$ linearly independent sets forces these sets to already be spanning.

Lemma 3.4. Let $\left\{f_{i}\right\}_{i \in I}$ be a set of vectors in an $N$-dimensional Hilbert space $\mathbb{H}_{N}$ and let $I_{j} \subset I, j=1,2, \ldots, r$, be linearly independent subsets. Assume that there is a partition of $I$ into $\left\{A_{j}\right\}_{j=1}^{r}$ so that

$$
\operatorname{span}\left\{f_{i}\right\}_{i \in A_{j}}=\mathbb{H}_{N}, \text { for all } j=1,2, \ldots, r \text {. }
$$

Then $\left\{I_{j}\right\}_{j=1}^{r}$ is a partition of $I$ and

$$
\operatorname{span}\left\{f_{i}\right\}_{i \in I_{j}}=\mathbb{H}_{N}, \text { for all } j=1,2, \ldots, r .
$$

Proof. For all $j=1,2, \ldots, r$, the fact that $\left\{f_{i}\right\}_{i \in I_{j}}$ are linearly independent implies that the dimension of the span of $\left\{f_{i}\right\}_{i \in I_{j}}$ is $\left|I_{j}\right|$. Also, the fact that $\left\{f_{i}\right\}_{i \in A_{j}}$ spans $\mathbb{H}_{N}$ implies $\left|A_{j}\right| \geq N$. Now, we have

$$
N r \geq \sum_{j=1}^{r} \operatorname{dim} \operatorname{span}\left\{f_{i}: i \in I_{j}\right\}=\sum_{j=1}^{r}\left|I_{j}\right|=|I|=\left|\bigcup_{j=1}^{r} A_{j}\right|=\sum_{j=1}^{r}\left|A_{j}\right| \geq N r .
$$

Hence, $\sum_{j=1}^{r} \operatorname{dim} \operatorname{span}\left\{f_{i}: i \in I_{j}\right\}=N r$ and $\sum_{j=1}^{r}\left|I_{j}\right|=|I|$. Thus,

$$
\operatorname{dim} \operatorname{span}\left\{f_{i}: i \in I_{j}\right\}=N
$$

for every $j=1,2, \ldots, r$, and the sets $\left\{I_{j}\right\}_{j=1}^{r}$ form a partition.

Next, we will give a reasonable lower bound on the number of spanning sets any frame contains in terms of the lower frame bound of the frame and the norms of the frame vectors.

Proposition 3.5. Let $\left\{f_{i}\right\}_{i \in I}$ be a frame for $\mathbb{H}_{N}$ with lower frame bound $A \geq 1$, let $\left\|f_{i}\right\|^{2} \leq 1$ for all $i \in I$ and set $r=\lfloor A\rfloor$. Then there exists a partition $\left\{I_{j}\right\}_{j=1}^{r}$ of I so that

$$
\operatorname{span}\left\{f_{i}: i \in I_{j}\right\}=\mathbb{H}_{N}, \text { for all } j=1,2, \ldots, r \text {. }
$$


In particular, the number of frame vectors in a unit norm frame with lower frame bound $A$ is greater than or equal to $\lfloor A\rfloor N$.

Proof. We replace $\left\{f_{i}\right\}_{i \in I}$ by $\left\{\frac{1}{\sqrt{r}} f_{i}\right\}_{i \in I}$ so that our frame has lower frame bound greater than or equal to 1 and $\left\|f_{i}\right\|^{2} \leq \frac{1}{r}$, for all $i \in I$. Assume that the frame operator for $\left\{f_{i}\right\}_{i \in I}$ has eigenvectors $\left\{e_{j}\right\}_{j=1}^{N}$ with respective eigenvalues $\lambda_{1} \geq \lambda_{2} \geq$ $\ldots \geq \lambda_{N} \geq 1$. We proceed by induction on $N$.

We first consider $N=1$. Since

$$
\sum_{i \in I}\left\|f_{i}\right\|^{2} \geq 1 \text { and }\left\|f_{i}\right\|^{2} \leq \frac{1}{r}
$$

it follows that $\left|\left\{i \in I: f_{i} \neq 0\right\}\right| \geq r$, and so we have a partition into $r$ spanning sets.

Next, we assume that the inductive hypothesis holds for any Hilbert space of dimension $N$ and let $\mathbb{H}_{N+1}$ be a Hilbert space of dimension $N+1$.

We check two cases:

Case I. Suppose there exists a partition $\left\{I_{j}\right\}_{j=1}^{r}$ of $I$ so that $\left\{f_{i}\right\}_{i \in I_{j}}$ is linearly independent for all $j=1,2, \ldots, r$.

In this case,

$$
N+1 \leq(N+1) \lambda_{N} \leq \sum_{j=1}^{N+1} \lambda_{j}=\sum_{i \in I}\left\|f_{i}\right\|^{2} \leq|I| \frac{1}{r},
$$

and hence

$$
|I| \geq r(N+1)
$$

However, by linear independence, we have

$$
|I|=\sum_{j=1}^{r}\left|I_{j}\right| \leq r(N+1) .
$$

Thus, $\left|I_{j}\right|=N+1$ for every $j=1,2, \ldots, r$, and so $\left\{f_{i}\right\}_{i \in I_{j}}$ are all spanning.

Case II. Our family cannot be partitioned into $r$ linearly independent sets. In this case, let $\left\{I_{j}\right\}_{j=1}^{r}$ and a subspace $\emptyset \neq S \subset \mathbb{H}_{N+1}$ be given by Theorem 3.2. If $S=\mathbb{H}_{N+1}$, we are done. Otherwise, let $P$ be the orthogonal projection onto the subspace $S$. Let

$$
I_{j}^{\prime}=\left\{i \in I_{j}: f_{i} \notin S\right\}, \quad I^{\prime}=\bigcup_{j=1}^{r} I_{j}^{\prime} .
$$

Theorem 3.2(c) implies that $\left\{(I-P) f_{i}\right\}_{i \in I_{j}^{\prime}}$ is linearly independent for all $j=$ $1,2, \ldots, r$. To see this, note that the non-zero elements of $\left\{(I-P) f_{i}\right\}_{i \in I}$ are $\left\{(I-P) f_{i}\right\}_{i \in I^{\prime}}$. Fix $1 \leq j \leq r$ and assume there are scalars $\left\{\alpha_{i}\right\}_{i \in I_{j}^{\prime}}$ with $\sum_{i \in I_{j}^{\prime}} \alpha_{i}(I-P) f_{i}=0$.

This implies by Theorem 3.2 (c) that $\sum_{i \in I_{j}^{\prime}} \alpha_{i} f_{i} \in S$, and so $\alpha_{i}=0$ for all $i \in I_{j}^{\prime}$.

Now, $\left\{(I-P) f_{i}\right\}_{i \in I^{\prime}}$ has a lower frame bound 1 in $(I-P)\left(\mathbb{H}_{N+1}\right)$, dim $(I-$ $P)\left(\mathbb{H}_{N+1}\right) \leq N$ and $\left\|(I-P) f_{i}\right\|^{2} \leq\left\|f_{i}\right\|^{2} \leq \frac{1}{r}$ for all $i \in I^{\prime}$.

Applying the induction hypothesis, we can find a partition $\left\{A_{j}\right\}_{j=1}^{r}$ of $I^{\prime}$ with span $\left\{(I-P) f_{i}\right\}_{i \in A_{j}}=(I-P)\left(\mathbb{H}_{N+1}\right)$ for all $j=1,2, \ldots, r$. 
Now, we can apply Lemma 3.4 together with the partition $\left\{A_{j}\right\}_{j=1}^{r}$ to conclude span $\left\{(I-P) f_{i}\right\}_{i \in I_{j}^{\prime}}=(I-P)\left(\mathbb{H}_{N+1}\right)$, and hence

$$
\operatorname{span}\left\{f_{i}\right\}_{i \in I_{j}}=\operatorname{span}\left\{S,\left\{(I-P) f_{i}\right\}_{i \in I_{j}^{\prime}}\right\}=\mathbb{H}_{N+1} \text {. }
$$

Note that we cannot expect to get any linear independence in Proposition 3.5 because our vectors can have arbitrarily small norms, and hence there can be an arbitrarily large number of them. However, we can remove appropriate vectors from the last $r-1$ sets until they are linearly independent and spanning. Putting the removed vectors into the first set, we get a partition into a spanning set and $r-1$ linearly independent spanning sets.

As an immediate consequence, we have the main result of this section.

Corollary 3.6. Let $\left\{f_{i}\right\}_{i \in I}$ be a Parseval frame for a finite dimensional Hilbert space $\mathbb{H}$ and let $r$ be a natural number so that $\left\|f_{i}\right\|^{2} \leq \frac{1}{r}$ for every $i \in I$. Then there is a partition $\left\{I_{j}\right\}_{j=1}^{r}$ of I so that

$$
\operatorname{span}\left\{f_{i}: i \in I_{j}\right\}=\mathbb{H}, \text { for all } j=1,2, \ldots, r .
$$

3.3. Partitioning a frame into linearly independent spanning sets. In this subsection, we want to combine the results from the previous two subsections to get a partition of a frame into linearly independent spanning sets. This will require a refinement of the proof of the generalization of the Rado-Horn Theorem given in [5. To help explain why we need such technical lemmas, let us first look at the main result we want to prove.

Theorem 3.7. Let $\left\{f_{i}\right\}_{i \in I}$ be a finite collection of vectors in a finite dimensional vector space $X$. Assume:

(1) $\left\{f_{i}\right\}_{i \in I}$ can be partitioned into $r+1$-linearly independent sets, and

(2) $\left\{f_{i}\right\}_{i \in I}$ can be partitioned into a set and $r$ linearly independent spanning sets.

Then there is a partition $\left\{I_{i}\right\}_{i=1}^{r+1}$ so that $\left\{f_{j}\right\}_{j \in I_{i}}$ is a linearly independent spanning set for all $i=2,3, \ldots, r+1$ and $\left\{f_{i}\right\}_{i \in I_{1}}$ is a linearly independent set.

The idea of the proof of Theorem 3.7 is the following. We will choose the partition of our frame into $r$ linearly independent spanning sets so that the dimension of the span of the remaining set is a maximum. Then, we will show that this remaining set must be linearly independent. This will be done by showing that if this remaining set is not linearly independent we can get a contradiction to the Rado-Horn Theorem. However, to get this contradiction will require a delicate interchange of the vectors from the sets in the partition. It is this interchange result that we will now develop.

We will be considering partitions which maximize dimensions in a very particular way.

Definition 3.8. Let $\left\{f_{i}\right\}_{i \in I}$ be a family of vectors. We say that a partition $\left\{I_{1}, \ldots, I_{M}\right\}$ of the index set $I$ has the maximality property (MD) if whenever $\left\{J_{i}\right\}_{i=1}^{M}$ is any partition of $I$ satisfying that for all $1 \leq i \leq M$, dim span $\left\{f_{j}\right\}_{j \in J_{i}} \geq$ dim span $\left\{f_{j}\right\}_{j \in I_{i}}$, then $\operatorname{dim} \operatorname{span}\left\{f_{j}\right\}_{j \in I_{i}}=\operatorname{dim} \operatorname{span}\left\{f_{j}\right\}_{j \in J_{i}}$ for all $i=1,2, \ldots$, $M$.

A straightforward consequence of maximality is the following:

Lemma 3.9. Let $\mathcal{F}=\left\{f_{i}: i \in I\right\}$ be a finite collection of vectors in a vector space. Let $M \in \mathbb{N}$ and $\left\{I_{j}: j=1, \ldots, M\right\}$ be a partition of I satisfying property (MD). If $f_{k} \in I_{p}$ and $f_{k}=\sum_{l \in I_{p}, l \neq k} \alpha_{l} f_{l}$, then $f_{k} \in \operatorname{span}\left(\mathcal{F}_{I_{j}}\right)$ for all $1 \leq j \leq M$. 
Proof. Assuming the hypothesis of the lemma, if $f_{k}=\sum_{l \in I_{p}, l \neq k} \alpha_{l} f_{l}$, then removing $f_{k}$ from $I_{p}$ keeps $\operatorname{dim} \operatorname{span}\left(\mathcal{F}_{I_{p}}\right)$ constant. By property (MD), moving $f_{k}$ into another $I_{j}, j \neq p$, cannot increase $\operatorname{dim} \operatorname{span}\left(\mathcal{F}_{I_{j}}\right)$, and the result follows.

If there are linearly dependent sets in a partition having property (MD), then we can move suitable vectors from one set to another. The following definition will be used to help us keep track of which vectors are being moved.

Definition 3.10. Let $\left\{f_{i}: i \in I\right\}$ be a collection of vectors in a vector space and let $\left\{I_{j}: j=1, \ldots, M\right\}$ be a partition of $I$. We define a chain of length one to be a set $\{(a, b)\}$ with $a \in I_{b}, b \in\{1,2, \ldots, M\}$ and $f_{a}=\sum_{j \in I_{b}, j \neq a} \alpha_{j} f_{j}$ for some choice of constants $\left\{\alpha_{j}\right\}_{j \in I_{b}, j \neq a}$. We define a chain of length $n$ to be a finite sequence $\left\{\left(a_{1}, b_{1}\right), \ldots,\left(a_{n}, b_{n}\right)\right\}$, where $a_{i} \in I$ and $b_{i} \in\{1, \ldots, M\}$, such that

- $\left(a_{1}, b_{1}\right)$ is a chain of length one,

- for $2 \leq i \leq n, a_{i} \in I_{b_{i}}$ and $f_{a_{i}}=\alpha f_{a_{i-1}}+\sum_{j \in I_{b_{i}}, j \neq a_{i}} \alpha_{j} f_{j}$ for some $\alpha \neq 0$, and

- $a_{i} \neq a_{k}$ for $i \neq k$.

A chain of length $n$ starting with $a_{1} \in L \subset I$ and ending at $a_{n} \in I$ is a chain of minimal length starting in $L$ and ending at $a_{n}$ if every chain starting in $L$ and ending at $a_{n}$ has length greater than or equal to $n$.

We recall the following lemma.

Lemma 3.11 (Casazza, Kutyniok, Speegle). Let $\left\{f_{i}: i \in I\right\}$ be a collection of vectors in a vector space, let $\left\{I_{j}: j=1, \ldots, M\right\}$ be a partition of $I$, and let $L \subset I_{1}$.

If $\left\{\left(a_{1}, b_{1}\right), \ldots,\left(a_{n}, b_{n}\right)\right\}$ is a chain of minimal length starting in $L$ and ending at $a_{n}$, then for each $1 \leq i \leq n,\left\{\left(a_{1}, b_{1}\right), \ldots,\left(a_{i}, b_{i}\right)\right\}$ is a chain of minimal length starting in $L$ and ending at $a_{i}$.

Proof. By induction it suffices to show that $\left\{\left(a_{1}, b_{1}\right), \ldots,\left(a_{n-1}, b_{n-1}\right)\right\}$ is a chain of minimal length. Suppose, for the sake of contradiction, that there did exist a chain $\left\{\left(u_{1}, v_{1}\right), \ldots,\left(u_{k}, v_{k}\right)\right\}$ such that $u_{k}=a_{n-1}$ and $k<n-1$. Since $\left\{\left(a_{1}, b_{1}\right), \ldots,\left(a_{n}, b_{n}\right)\right\}$ is a chain,

$$
f_{a_{n}}=\alpha f_{a_{n-1}}+\sum_{j \in I_{b_{n}}, j \neq a_{n}} \alpha_{j} f_{j}
$$

for some $\alpha \neq 0$. Therefore, either $\left\{\left(u_{1}, v_{1}\right), \ldots,\left(u_{k}, v_{k}\right),\left(a_{n}, b_{n}\right)\right\}$ is a chain with length $k+1<n$ or $a_{n}=u_{i}$ for some $i \leq k$, either of which contradicts the minimality of $n$.

Next, we show how to choose a partition having property (MD).

Lemma 3.12. Let $\mathcal{F}=\left\{f_{i}: i \in I\right\}$ be a finite collection of vectors, and let $M \in \mathbb{N}$. There exists among all the partitions of $I$ into $M$ non-empty subsets a partition $\left\{I_{1}, I_{2}, \ldots I_{M}\right\}$ with the property $(M D)$. This partition can be chosen so that $\mathcal{F}_{I_{j}}$ is linearly independent for all $2 \leq j \leq M$.

Proof. The set of partitions of $I$ into $M$ sets has a partial ordering with respect to which two partitions $\left\{I_{j}\right\}_{j=1}^{M}$ and $\left\{J_{j}\right\}_{j=1}^{M}$ satisfy $\left\{I_{j}\right\}_{j=1}^{M} \leq\left\{J_{j}\right\}_{j=1}^{M}$ if $\operatorname{dim} \mathcal{F}_{I_{j}} \geq$ $\operatorname{dim} \mathcal{F}_{J_{j}}$ for all $j \in\{1,2, \ldots, M\}$. I is a finite set, so there are maximal elements. By definition, these partitions have the property (MD). 
Assume that there is a partition with property (MD) which contains more than one set for which the associated vectors are linearly dependent, say $I_{1}$ and $I_{2}$. We can then successively remove indices from $I_{2}$ and place them into $I_{1}$ if the associated vectors are linear combinations of others remaining in the set indexed by $I_{2}$. After finitely many such moves, $\mathcal{F}_{I_{2}}$ is linearly independent. Moreover, by Lemma 3.9. the span of $\mathcal{F}_{I_{1}}$ and $\mathcal{F}_{I_{2}}$ retain their dimensions, which means the maximality is preserved.

If $\mathcal{F}_{I_{2}}, \ldots, \mathcal{F}_{I_{M}}$ are linearly independent, $L=\left\{i \in I_{1}: f_{i}=\sum_{j \in I_{1}, j \neq i} \alpha_{j} f_{j}\right\}$, and $\left\{\left(a_{1}, b_{1}\right), \ldots,\left(a_{n}, b_{n}\right)\right\}$ is a chain of minimal length starting in $L$, it follows that for each $1 \leq i<n, b_{i} \neq b_{i+1}$. In this case, we can track the changes in the partition as vectors are moved among the sets in a straightforward manner.

Definition 3.13. If $\mathcal{F}_{I_{2}}, \ldots, \mathcal{F}_{I_{M}}$ are linearly independent, then proceeding by induction, we can define

$$
U_{k}^{1}=I_{k}, \quad 1 \leq k \leq M
$$

and for $2 \leq i \leq n$,

$$
\begin{aligned}
U_{k}^{i} & =U_{k}^{i-1} \text { for } k \neq b_{i-1}, k \neq b_{i}, \\
U_{b_{i}}^{i} & =U_{b_{i}}^{i-1} \cup\left\{a_{i-1}\right\}, \\
U_{b_{i-1}}^{i} & =U_{b_{i-1}}^{i-1} \backslash\left\{a_{i-1}\right\} .
\end{aligned}
$$

Lemma 3.14. Let $\mathcal{F}=\left\{f_{i}: i \in I\right\}$ be a finite collection of vectors, and let $\left\{I_{1}, I_{2}\right.$, $\left.\ldots, I_{M}\right\}$ be a partition with the property $(M D)$ for which $\mathcal{F}_{I_{2}}, \ldots, \mathcal{F}_{I_{M}}$ are linearly independent. Let $L$ be as above and assume that $\left\{\left(a_{1}, b_{1}\right), \ldots,\left(a_{n}, b_{n}\right)\right\}$ is a minimal chain starting in $L$. For each $1 \leq i \leq n, f_{a_{i}}$ can then be written as the sum

$$
f_{a_{i}}=\sum_{j \in I_{b_{i}}, j \notin\left\{a_{p}: 1 \leq p \leq n\right\}} \alpha_{j} f_{j}+\sum_{j \in U_{b_{i}}^{i} \cap\left\{a_{p}: 1 \leq p<i\right\}} \alpha_{j} f_{j} .
$$

Proof. For the case $i=1$, note that $a_{1} \in L$ implies that $f_{a_{1}}=\sum_{j \in L, j \neq a_{1}} \alpha_{j} f_{j}$ for some choice of $\alpha_{j}$. By Lemma 3.11 none of these $j \in L$ can be in $\left\{a_{p}: 1 \leq p \leq n\right\}$ since this would not be a chain of minimal length starting in $L$. Recalling that $b_{i}=1$, we prove the claim for $i=1$.

Proceeding by induction, let $i \in\{1, \ldots, n\}$, and we assume (3.2) is true for $1 \leq k<i$. We will show that it is also true for $i$. Note that

$$
\begin{aligned}
f_{a_{i}} & =\alpha f_{a_{i-1}}+\sum_{j \in I_{b_{i}}, j \neq a_{i}} \alpha_{j} f_{j} \\
& =\alpha f_{a_{i-1}}+\sum_{j \in I_{b_{i}} \cap U_{b_{i}}^{i}, j \neq a_{i}} \alpha_{j} f_{j}+\sum_{j \in I_{b_{i}} \backslash U_{b_{i}}^{i}} \alpha_{j} f_{j} \\
& =\alpha f_{a_{i-1}}+\sum_{j \in I_{b_{i}} \cap U_{b_{i}}^{i}, j \neq a_{i}} \alpha_{j} f_{j}+\sum_{j \in I_{b_{i}} \cap\left\{a_{p}: 1 \leq p<i-1\right\}} \alpha_{j} f_{j},
\end{aligned}
$$

where we have used in the last two lines that $I_{b_{i}} \cap\left\{a_{p}: 1 \leq p<i-1\right\}=I_{b_{i}} \backslash U_{b_{i}}^{i}$. Now, suppose for the sake of contradiction that there is a $j \in I_{b_{i}} \cap U_{b_{i}}^{i}$ such that $\alpha_{j} \neq 0$ and $j=a_{p}$ for some $p>i$. Then $\left\{\left(a_{1}, b_{1}\right), \ldots,\left(a_{i-1}, b_{i-1}\right),\left(a_{p}, b_{i}\right)\right\}$ is a chain starting in $L$, which contradicts the minimality of the chain $\left\{\left(a_{1}, b_{1}\right), \ldots,\left(a_{n}, b_{n}\right)\right\}$. 
Thus, using the induction hypothesis on each term in the last sum in (3.4) and combining terms, one obtains

$$
f_{a_{i}}=\alpha f_{a_{i-1}}+\sum_{j \in I_{b_{i}}, j \notin\left\{a_{p}: 1 \leq p \leq n\right\}} \tilde{\alpha}_{j} f_{j}+\sum_{j \in U_{b_{i}}^{i} \cap\left\{a_{p}: 1 \leq p<i\right\}} \tilde{\alpha}_{j} f_{j}
$$

with an appropriate choice of $\tilde{\alpha}_{j}$ 's.

Finally, we will identify the fundamental property needed from a chain of maximal length.

Lemma 3.15. Let $\mathcal{F}=\left\{f_{i}: i \in I\right\}$ be a finite collection of vectors and $\left\{I_{j}\right\}_{j=1}^{M}$ be a partition of the index set I into $M \in \mathbb{N}$ non-empty sets which has the property (MD) and for which sets $I_{2}, I_{3}, \ldots, I_{M}$ index linearly independent sets. Moreover, let $L=\left\{i \in I_{1}: f_{i}=\sum_{j \in I_{1}, j \neq i} \alpha_{j} f_{j}\right\}, L_{0}=\{i \in I$ : there is a chain starting in $L$ and ending at $i\}$, and $L_{j}=L_{0} \cap I_{j}$ for $1 \leq j \leq M$. If $\left\{\left(a_{1}, b_{1}\right), \ldots,\left(a_{n}, b_{n}\right)\right\}$ is a chain of minimal length starting in $L$ and ending at $a_{n}$, then $f_{a_{n}} \in \operatorname{span}\left(\mathcal{F}_{L_{m}}\right)$ for all $1 \leq m \leq M$.

Proof. We show that if $\left\{\left(a_{1}, b_{1}\right), \ldots,\left(a_{n}, b_{n}\right)\right\}$ is a chain of minimal length starting in $L$ and ending at $a_{n}$, then $f_{a_{n}} \in \operatorname{span}\left(\mathcal{F}_{L_{m}}\right)$ for each $1 \leq m \leq M$.

For $n=1$, fix $m \in\{1, \ldots, M\}$, and observe that $a_{1} \in L$. Hence, by Lemma 3.9, we can write $f_{a_{1}}=\sum_{l \in I_{m}} \alpha_{l} f_{l}$. For each $l$ such that $\alpha_{l} \neq 0,\left(a_{1}, 1\right),(l, m)$ is a chain ending at $l$. Therefore, $f_{a_{1}} \in \operatorname{span}\left(\mathcal{F}_{L_{m}}\right)$, as desired.

By Lemma 3.14 and the fact that $I_{b_{i}} \backslash\left\{a_{p}: 1 \leq p \leq n\right\} \subset U_{b_{i}}^{k}$ for all $1 \leq k \leq n$, we have that $f_{a_{i}} \in \operatorname{span}\left(\mathcal{F}_{U_{b_{i}}^{i} \backslash\left\{a_{i}\right\}}\right)$. Therefore, $\operatorname{dim} \operatorname{span}\left(\mathcal{F}_{U_{b_{i}}^{i}}\right)=\operatorname{dim} \operatorname{span}\left(\mathcal{F}_{U_{b_{i}}^{i+1}}\right)$. In particular, the partition $\left\{U_{k}^{i}: 1 \leq k \leq M\right\}$ satisfies property (MD).

By property (MD), Lemma 3.14, and Lemma 3.9, $f_{a_{n}} \in \operatorname{span}\left(\mathcal{F}_{U_{m}^{n}}\right)$ for each $1 \leq m \leq M$. Therefore, for $m \neq b_{n}$, there exist $\alpha_{j}^{(0)}$ such that

$$
\begin{aligned}
f_{a_{n}} & =\sum_{j \in U_{m}^{n}} \alpha_{j}^{(0)} f_{j}=\sum_{j \in U_{m}^{n} \cap I_{m}} \alpha_{j}^{(0)} f_{j}+\sum_{j \in U_{m}^{n} \backslash I_{m}} \alpha_{j}^{(0)} f_{j} \\
& =\sum_{j \in U_{m}^{n} \cap I_{m}} \alpha_{j}^{(0)} f_{j}+\sum_{j \in\left\{a_{p}: b_{p+1}=m, 1 \leq p<n-1\right\}} \alpha_{j}^{(0)} f_{j} .
\end{aligned}
$$

By definition of a chain, for each $a_{p}$ such that $b_{p+1}=m$ and $1 \leq p<n-1$,

$$
f_{a_{p}}=\alpha^{p} f_{a_{p+1}}+\sum_{j \in I_{m}, j \neq a_{p+1}} \alpha_{j}^{(p)} f_{j},
$$

for some choice of $\alpha_{j}^{(p)}$ and some $\alpha^{(p)} \neq 0$.

Fix $j_{0}$ such that $\alpha_{j_{0}}^{(0)} \neq 0$ in (3.5). We show that $j_{0} \in L_{m}$, which finishes the proof of the lemma. Clearly, if $j_{0} \in\left\{a_{1}, \ldots, a_{n}\right\}$, then we are done, so we assume that $j_{0} \notin\left\{a_{1}, \ldots, a_{n}\right\}$.

Case 1 . There is some $1 \leq p<n-1$ such that $b_{p+1}=m$ and $\alpha_{j_{0}}^{(p)} \neq 0$. Then, one can solve (3.6) for $f_{j_{0}}$ to obtain

$$
f_{j_{0}}=\beta f_{a_{p}}+\sum_{j \in I_{m}, j \neq j_{0}, j \neq a_{p}} \beta_{j} f_{j}
$$

for some $\beta \neq 0$. Hence, $\left(a_{1}, b_{1}\right), \ldots,\left(a_{p}, b_{p}\right),\left(j_{0}, m\right)$ is a chain and $j_{0} \in L_{m}$. 
Case 2. For each $1 \leq p<n-1$ such that $b_{p+1}=m$, we have $\alpha_{j_{0}}^{(p)}=0$. We have

$$
\begin{aligned}
f_{a_{n}} & =\sum_{j \in U_{m}^{n} \cap I_{m}} \alpha_{j}^{(0)} f_{j}+\sum_{j \in\left\{a_{p}: b_{p+1}=m, 1 \leq p<n-1\right\}} \alpha_{j}^{(0)} f_{j} \\
& =\sum_{j \in U_{m}^{n} \cap I_{m}} \alpha_{j}^{(0)} f_{j}+\sum_{p \in\left\{p: b_{p+1}=m, 1 \leq p<n-1\right\}} \alpha_{a_{p}}^{(0)} f_{a_{p}} \\
& =\sum_{j \in U_{m}^{n} \cap I_{m}} \alpha_{j}^{0} f_{j}+\sum_{p \in\left\{p: b_{p+1}=m, 1 \leq p<n-1\right\}} \alpha_{a_{p}}^{(0)}\left(\alpha^{(p)} f_{a_{p+1}}+\sum_{j \in I_{m}, j \neq a_{p+1}} \alpha_{j}^{(p)} f_{j}\right) \\
& =\alpha_{j_{0}}^{(0)} f_{j_{0}}+\sum_{j \in I_{m}, j \neq j_{0}} \tilde{\alpha}_{j} f_{j},
\end{aligned}
$$

where the first equality is (3.5), the second equality is a re-indexing, the third equality follows from (3.6), and the last equality holds for some choice of $\tilde{\alpha}_{j}$ by combining sums, since $\alpha_{j_{0}}^{(p)}=0$ for all $1 \leq p<n-1$ such that $b_{p+1}=m$, and $j_{0} \notin\left\{a_{1}, \ldots, a_{n}\right\}$. Therefore, $\left\{\left(a_{1}, b_{1}\right), \ldots,\left(a_{n}, b_{n}\right),\left(j_{0}, m\right)\right\}$ is a chain and $j_{0} \in L_{m}$.

Now we can prove the main result of this subsection.

Proof of Theorem 3.7. We choose the partition $\left\{I_{i}\right\}_{i=1}^{r+1}$ of $I$ that maximizes dim span $\left\{f_{j}\right\}_{j \in I_{1}}$ taken over all partitions so that the last $r$ sets span $X$. If $\left\{J_{i}\right\}_{i=1}^{r+1}$ is a partition of $I$ such that for all $1 \leq i \leq r+1$, dim span $\left\{f_{j}\right\}_{j \in J_{i}} \geq \operatorname{dim}$ span $\left\{f_{j}\right\}_{j \in I_{i}}$, then $\operatorname{dim} \operatorname{span}\left\{f_{j}\right\}_{j \in I_{i}}=\operatorname{dim} \operatorname{span}\left\{f_{j}\right\}_{j \in J_{i}}$ for all $i=2, \ldots, r+1$, since dim span $\left\{f_{j}\right\}_{j \in I_{i}}=\operatorname{dim} X$, and $\operatorname{dim} \operatorname{span}\left\{f_{j}\right\}_{j \in I_{1}}=\operatorname{dim} \operatorname{span}\left\{f_{j}\right\}_{j \in J_{1}}$ by construction. This means the chosen partition has the property (MD) and the properties asserted by Lemma 3.15. Suppose that this does not partition $\mathcal{F}_{I}$ into linearly independent sets, i.e. $\mathcal{F}_{I_{1}}$ is not linearly independent. As in Lemma 3.15, let $L=\left\{i \in I_{1}: f_{i}=\sum_{j \in I_{1}, j \neq i} \alpha_{j} f_{j}\right\}$ be the index set of the "linearly dependent vectors" in $I_{1}, L_{0}=\{i \in I$ : there is a chain starting in $L$ and ending at $i\}$, and $L_{j}=L_{0} \cap I_{j}, 1 \leq j \leq r+1$.

Let $S=\operatorname{span}\left(\mathcal{F}_{L_{0}}\right)$. By Lemma 3.15, $S=\operatorname{span}\left(\mathcal{F}_{L_{j}}\right)$ for all $1 \leq j \leq r+1$. Moreover, for $1 \leq j \leq r+1, i \in L_{j}$ implies that $i \in I_{j}$ and $f_{i} \in S$. Therefore,

$$
S \subset \operatorname{span}\left\{f_{i}: i \in L_{j}\right\} \subset \operatorname{span}\left\{f_{i}: i \in I_{j}, f_{i} \in S\right\}=S .
$$

Let $J=\left\{i \in I: f_{i} \in S\right\}$. By construction, $L \subset J$. Let $d=\operatorname{dim}(S)$ and see that, by the preceding portion of this proof, $\operatorname{dim} \operatorname{span}\left(\mathcal{F}_{J}\right)=d$. Moreover,

$$
|J|=\left|L_{1}\right|+\cdots+\left|L_{M}\right|=\left|L_{1}\right|+r d>d(r+1),
$$

because $L_{1}$ is linearly dependent, since it contains $L$ by virtue of chains of length one. Therefore, for $J=\left\{i \in I: f_{i} \in S\right\}$, $\frac{|J|}{\operatorname{dimspan}\left(\mathcal{F}_{J}\right)}>r+1$. This is in contradiction with assumption (1), which implies by the Rado Horn theorem that $|J| / d \leq r+1$ and thus completes the proof of the theorem.

\section{ACKNowledgments}

The authors would like to thank the American Institute of Mathematics for its hospitality and the support of the Structured Quartet Research Ensemble on the Kadison Singer Problem. A special thanks to the referee for detailed and thoughtful suggestions which significantly improved the presentation of this paper. 


\section{REFERENCES}

[1] J. Anderson, Extreme points in sets of positive linear maps on B(H), J. Funct. Anal. 31 (1979), 303-329. MR.525951 (80k:46070)

[2] B. G. Bodmann, P. G. Casazza and G. Kutyniok, A quantitative notion of redundancy for finite frames, Appl. Comput. Harmon. Anal. 30 (2011), no. 3, 348-362. MR2784569

[3] P. Casazza, O. Christensen, A. Lindner and R. Vershynin, Frames and the Feichtinger conjecture, Proc. Amer. Math. Soc. 133 (2005), no. 4, 1025-1033. MR2117203(2006a:46024)

[4] P. G. Casazza, M. Fickus, E. Weber and J. C. Tremain, The Kadison-Singer Problem in mathematics and engineering - A detailed account, Contemp. Math. 414, Operator theory, operator algebras and applications, D. Han, P. E. T. Jorgensen and D. R. Larson, eds., Amer. Math. Soc., Providence, RI (2006), 297-356. MR2277219 (2007j:42016)

[5] P. G. Casazza, G. Kutyniok, and D. Speegle, A redundant version of the Rado-Horn Theorem, Linear Algebra Appl. 418 (2006), 1-10. MR2257571 (2007h:15003)

[6] P. G. Casazza, G. Kutyniok, D. Speegle and J. C. Tremain, A decomposition theorem for frames and the Feichtinger conjecture, Proc. Amer. Math. Soc. 136 (2008), 2043-2053. MR2383510 (2009f:46032)

[7] P. G. Casazza and J. Tremain, The Kadison-Singer problem in mathematics and engineering, Proc. Nat. Acad. of Sciences 103, no. 7 (2006), 2032-2039. MR2204073 (2006j:46074)

[8] O. Christensen, An introduction to frames and Riesz bases, Birkhäuser, Boston (2003). MR $1946982(2003 \mathrm{k}: 42001)$

[9] I. Daubechies, From the original framer to present-day time-frequency and time-scale frames, J. Fourier Anal. Appl. 3(5) (1997), 485-486. MR.1491928

[10] I. Daubechies and B. Han, The canonical dual frame of a wavelet frame, Appl. Comput. Harmon. Anal. 12(3) (2002), 269-285. MR.1912147 (2003i:42050)

[11] I. Daubechies, B. Han, A. Ron and Z. Shen, Framelets: MRA-based constructions of wavelet frames, Appl. Comput. Harmon. Anal. 14(1) (2003), 1-46. MR.1971300 (2004a:42046)

[12] J. Edmonds and D. R. Fulkerson, Transversals and matroid partition, J. Res. Nat. Bur. Standards Sect. B 69B (1965), 147-153. MR0188090 (32:5531)

[13] K. Gröchenig, Foundations of time-frequency analysis, Birkhäuser, Boston (2000). MR:1843717 (2002h:42001)

[14] A. Horn, A characterization of unions of linearly independent sets, J. London Math. Soc. 30 (1955), 494-496. MR0071487 (17:135d)

[15] J. Kovačević and A. Chebira, Life beyond bases: The advent of frames (Part I), IEEE Signal Proc. Mag. 24(4) (2007), 86-104.

[16] J. Kovačević and A. Chebira, Life beyond bases: The advent of frames (Part II), IEEE Signal Proc. Mag. 24(5) (2007), 115-125.

[17] J. Oxley, Matroid theory, Oxford University Press, New York (2006). MR1207587 (94d:05033)

[18] R. Rado, A combinatorial theorem on vector spaces, J. London Math. Soc. 37 (1962), 351353. MR0146186 (26:3708)

[19] Amos Ron and Zuowei Shen, Affine systems in $L_{2}\left(\mathbb{R}^{d}\right)$ : The analysis of the analysis operator, J. Funct. Anal. 148(2) (1997), 408-447. MR1469348 (99g:42043)

Department of Mathematics, 651 Philip G. Hoffman Hall, University of Houston, Houston, Texas 77204-3008

E-mail address: bgb@math.uh.edu

Department of Mathematics, University of Missouri, Columbia, Missouri 65211

E-mail address: casazzap@missouri.edu

Department of Mathematics, 651 Philip G. Hoffman Hall, University of Houston, Houston, Texas 77204-3008

E-mail address: vern@math.uh.edu

Department of Mathematics and Computer Science, Saint Louis University, St. Louis, Missouri 63103

E-mail address: speegled@slu.edu 